\title{
CHEMICAL ABUNDANCES AND CHEMICAL EVOLUTION OF THE MAGELLANIC CLOUDS: PROSPECTS FOR THE FUTURE
}

\author{
M.A. DOPITA \\ Mt. Stromlo and Siding Spring Observatories, \\ The Australian National University.
}

\begin{abstract}
The prospects for future advances in understanding the chemical evolution of the Magellanic Clouds is reviewed on the basis of results presented at IAU Symposium \#148. These are divided into three areas, the metallicity - age relationships, the current abundance set, and the theory of the chemical evolution of the Clouds.
\end{abstract}

\section{Introduction}

The problem of the chemical evolution of the Magellanic Clouds resolves itself into three main areas. First, what was the history of the chemical enrichment of the Magellanic Clouds? Second, what is the current abundance set in the ISM of the clouds, and is it uniform? Third, how and why does the chemical enrichment differ from the Galaxy, and why is this so? In this review, I will briefly summarise what I have learnt from this conference, and what future observations are suggested by these insights. Contributions presented in this conference are referred to by their first authors, whereas other references are given more conventionally.

\section{The Metallicity-Age Relationships}

During the conference, we heard a great deal about the methods that have been used by Bob Schommer, Mike Bessell, Gary Da Costa and Doug Geisler to obtain the metallicity-age relationships for both clouds using observations of the clusters. These are based in isochrone and/or colour fitting to obtain the age, and using either line or blanketing fitting to derive the metallicity. Many years ago, Ben Gascoigne (1980) was telling us that the metallicity-age relationship was much flatter for the LMC than it had been for the local region of the Galaxy, and that, for the SMC, it was flatter still. It is very pleasing to see that these pioneering results have been so abundantly confirmed.

However, for every advance, more and new questions are raised. It is clear that we see in the LMC very few (or no) clusters made 3 - 13 Gyr ago. It is also clear that, at the same time, the LMC succeeded in enriching itself from an $[\mathrm{Fe} / \mathrm{H}\rceil \sim-2.0$ to a value of about -0.8 , It is certain, therefore, that stars were being made throughout this period. Without data in this age range, we can say very little about the details of the chemical enrichment process in the LMC. It is possible that the absence of clusters is simply a selection effect, and that such clusters as have been made have simply faded from view, or else have been disrupted by tidal influences. The search for the Type VII clusters being carried out by Gary Da Costa will be very important in the resolution of this problem.

In the case of the SMC we are faced with almost the inverse problem. We see clusters at all ages, but until about $5 \mathrm{Gyr}$ ago, the metallicity seemed to stay almost constant at about $[\mathrm{Fe} / \mathrm{H}] \sim-1.3$. Thus we are faced with the conundrum, how did the SMC make clusters without enriching the gas?

The Spites presented us with an alarming possibility, namely, that the clusters may have a different metallicity from the field. We clearly need observations of main sequence field stars to confirm this result, and to apply the same methodology in the Magellanic Clouds as Twarog (1980) used in the Galaxy. This should now be possible with the instrumentation 
that is available on modern telescopes.

Sydney van den Bergh presented us with an interesting question. Why is it that the young cluster around the Bars? The young clusters, that is! Did the formation of the Bars in the Magellanic Clouds encourage an enhanced rate of star formation in their vicinity, or is it simply that the Bars lie in the regions of the Magellanic Clouds which have greatest surface density, and in which the specific rate of star formation is therefore the highest.

It would be nice to have other observational methods to crack the nut of the age metallicity relationships. Two new possibilities have been raised in this conference. First, Peter Wood has shown us that the period - luminosity relationships for LPV stars appear to be metallicity dependent. If this can be made to work with sufficient accuracy, this would present a valuable new technique. Second, the studies of planetary nebulae (PN) (Mike Barlow, Mike Dopita) have shown that a real scatter in metallicity exists for the heavier $\alpha$ process elements which are not affected by the various chemical dredge-up episodes that afflict the AGB stars. For the LMC, I have shown that the abundance spread is as large as 1.0 dex in $[\mathrm{Ne} / \mathrm{H}]$ or in $[\mathrm{Ar} / \mathrm{H}]$. For the case of $\mathrm{Ne}$ we have to beware of the possiblity of the dredge-up of ${ }^{22} \mathrm{Ne}$. Nonetheless, these results imply that the LMC PN should cover an abundance range $-0.3 \approx[\mathrm{Fe} / \mathrm{H}] \gtrsim-1.6$, which in turn implies an age range of about $1-12$ Gyr. There is clear evidence that the metallicity is related directly to the core mass for both the Magellanic Clouds (Dopita, this conference), and for the PN in the Galactic Bulge (Dopita et al. 1990). Of course, the core mass is itself dependent on both initial metallicity and age, but we can hope that the self-consistent models being carried through by Vassiliadis and Wood (in preparation), will be able to resolve these components, enabling us to reconstruct the true age - metallicity relationships for the Clouds.

\section{The Current Abundance Set}

At this conference have heard presented a variety of new results on the current abundances in both Magellanic Clouds. For the heavier elements, the best diagnostic tool we have appears to be in the observation of the cooler supergiant stars (the Spites, Steve Russell, Mike Bessell). For the hotter stars, we get information on the lighter elements from spectra taken in the visible, but UV observations are needed for the heavier elements. Rolf-Peter Kudritski has shown us that (very complex) diagnostic tools to do this job have been developed by his group. However, using the most luminous OB stars in the Clouds, the abundances he derives so far are not in good agreement with the other methods, being systematically too low. It remains to be seen if this is real, and if so, what it is caused by. For example, Kahn (1974) has discussed the possibility that dust is pushed away from the star through the (metal depleted) gas during a cocoon phase of accretion.

Both the integrated spectra of HII regions and individual slit spectra on evolved SNR have been used to derive abundances in the ISM (Steve Russell, Mike Dopita). The SNR are particulary useful in giving the abundances of some of the heavier elements such as $\mathrm{Ca}, \mathrm{Fe}$ and $\mathrm{Ni}$, which can then be compared with the cool supergiants. So far it appears that the two abundance scales match well, so that we can be reasonably confident that we have derived a "global" abundance set. These results show that the [O/Fe] ratio in both clouds is lower than the Galactic value at the corresponding value of $[\mathrm{Fe} / \mathrm{H}]$. There appears to be a persisting problem with C, which comes out to be much lower in the HII regions, and, to a lesser extent, in the SNR. This raises the possibility that gas-phase depletion onto grains is important. This possibility is also raised by the UV interstellar line absorption studies presented by Klaas de Boer.

In view of these problems, the observations which we must try to obtain are obvious. First, we need a lot more observations of the lower luminosity main-sequence B-stars in which the non-LTE effects are not so extreme. I understand that the Munich, Heidelberg and Belfast groups have such an observational program underway. In order to settle the 
discrepancy between the HII region abundances and the $\mathrm{O}$ stars, we need to investigate both the abundances of HII regions and of their exciting stars. Modelling should be selfconsistent, in that the ionisation model of the HII region should use the theoretical UV spectrum of the central star for the derived abundance. Finally, we can confidently expect that HST will provide absorption line studies in lines of sight to OB stars to give us not only abundance data, but also a probe of the three-dimensional structure of both clouds.

\section{Chemical Evolution Theory.}

The observations presented in this conference present us with a variety of interesting facts to be explained by an improved theory of the chemical enrichment of the clouds. In no particular order these are:

How did the SMC stay so metal poor so long? There are three possibilities. Either it did not collapse until $5 \mathrm{Gyr}$ ago, or the rate of infall matched the rate of chemical enrichment rather well, or else it was losing its metals in a wind as fast as they were produced. The first of these is rather unlikely, since clusters at least were forming between 12 and $5 \mathrm{Gyr}$ ago. The second and the third possibilities, or some combination of them, are more likely.

What determines the current value of the $[\mathrm{O} / \mathrm{Fe}]$ ratio? We know that $\mathrm{O}$ is produced predominantly in the Type II supernovae. We also know that a little $\mathrm{Fe}$ is also produced in Type II supernovae. If SN 1987A (John Danziger) was typical, then the asymptotic value of the $[\mathrm{O} / \mathrm{Fe}]$ ratio in our halo $(\sim 0.6)$ can be explained as being set by Type II supernovae (Dopita et al. 1988). The majority of the Fe, however, is produced in Type Ia supernovae after some time delay, while the stars involved evolve to their pre-supernova configuration. Thus, the slope of the $[\mathrm{O} / \mathrm{Fe}]:[\mathrm{Fe} / \mathrm{H}]$ relationship at late times is set by either the infall timescale (Francesca Matteucci, reported by John Danziger), or by a steeper slope of the IMF ? (Russell and Dopita, 1990). To some extent, the results of Massey and Garmany reported here by Rob Kennicutt support the idea that the slope of the IMF is in fact steeper in the Magellanic Clouds.

Finally, we have the question of the role of the Bars in both clouds in influencing the star formation in, and chemical evolution of, the Magellanic Clouds. There seems to be little doubt that the past $\sim 4$ Gyr in the LMC and the SMC have seen a greatly enhanced rate of star formation. This may have been due to infall, but nontheless, the association of the young clusters with the regions of the Magellanic Clouds swept out by the Bars is very suggestive. We would like to know whether the tidal interaction between SMC and LMC triggered the recent star formation activity and enrichment in the Bars or, rather, did the formation of bars trigger star formation? For this, we require a much better understanding of the orbital parameters of the Clouds, and a theory which describes both the stellar and gas response to bar development.

\section{References}

Dopita, M.A., Dawe, J.A., Achilleos, N., Brissenden, R.J.V., Flynn, C., Meatheringham, S.J., Rawlings, S,. Tuohy, I.J., McNaught, R.D., Coates, D.W., Hancy, S., Thompson, K., Shobbroock, R.R. (1988), Astron. J. 95, 1717.

Dopita, M.A., Henry, J.P., Tuohy, I.R., Webster, B.L., Roberts, E.H., Byun, Y.-I., Cowie, L.L., Songaila, A. (1990), Astrophys. J. (in press).

Gascoigne, S.C.B. (1980), IAU Symp. 85 Star Clusters, J.E. Hesser (ed.) (Reidel: Dordrecht) p.305.

Kahn, F.D. (1974), The Interstellar Medium, K. Pinkau (ed.) (Reidel: Dordrecht) p.235.

Russell, M.A., Dopita, M.A. (1990), Astrophys. J. Suppl. Ser. (in press).

Twarog, B.A. (1980), Astrophys. J. 242, 242. 